\title{
sciendo
}

\section{THE ROLE OF ARGININE IN DISEASE PREVENTION, GUT MICROBIOTA MODULATION, GROWTH PERFORMANCE AND THE IMMUNE SYSTEM OF BROILER CHICKEN - A REVIEW}

\author{
Harry A. Aguzey, Zhenhua Gao*, Wu Haohao, Cheng Guilan, Wu Zhengmin, Chen Junhong, \\ Niu Zhi Li \\ Department of Animal Science, College of Agriculture, \\ Guangdong Ocean University, Zhanjiang, Guangdong 524088, P.R China \\ •Corresponding author: xmsgzhh@126.com
}

\begin{abstract}
The effect of dietary arginine on disease prevention, immune system modulation, the gut microbiota composition and growth of broiler chicken was reviewed. The main aim of poultry production is the maximization of profit at the least possible cost. This objective can mainly be achieved by ensuring that there is no interference in growth or disease outbreak and by feeding chicken with the best possible level of nutrients. With the ban on antibiotic growth promoters, attention is shifted towards other nutrition methods to prevent diseases and promote growth. More attention is therefore given to protein diets in animal nutrition due to their importance as essential part of active biological compounds in the body, assisting in the breakdown of body tissue and helping in the physiological processes of the animal. Arginine plays important function in serving as building blocks of proteins and polypeptides. It performs other roles during the regulation of important biochemical functions such as maintenance, growth, reproduction and immunity. Arginine cannot be synthesized by the body so it has to be supplemented in the diet. When arginine is supplemented above the recommended level, the gut mucosa is protected, immunosuppression is alleviated, diseases like necrotic enteritis, infectious bursal disease and coccidiosis in broiler chickens are prevented. There is an improvement in growth resulting from the increase in intestinal absorption, barrier function and microbiota composition.
\end{abstract}

Key words: arginine, immune system, growth modulator, microbiota, immune modulation

Protein requirement of farm animals ranges from 15 to $22 \%$, this underlines the importance of protein in the composition of animal diet since it serves as the basis for the development of the animal sector (NRC, 1994; Ravindran, 2016). In terms of production, it accounts for a great percentage of the total cost of feed but cannot be done away with since it is an important nutrient for growth performance of livestock and poultry. 
Amino acids are nutrients that are indispensable in the poultry industry. In the nutrition of chicken, one major nutrient worthy of note is protein and by extension amino acids. Arginine being one such important amino acid is rated as essential because it is needed as a foundation for proteins and polypeptides. The requirement of arginine by birds far outweighs that of other researched species (Ball et al., 2007). Arginine and its products (polyamines, nitric oxide, agmatine) perform important immune and biochemical roles in birds (Khajali and Wideman, 2010). It performs roles such as the regulation of crucial pathways of metabolism which are important for physiological processes; body maintenance, growth, reproduction and immunity (Liu et al., 2012 b; Wu et al., 2012). Arginine is an important amino acid needed by poultry but cannot be produced by the urea cycle due to absence of carbamoyl phosphate synthetase I in mitochondria (Lewis, 1996). For this reason, it needs to be supplied in the diet of chicken to be able to perform its various functions (Khajali and Wideman, 2010).

According to the NRC (1994), the requirement of arginine for starters, growers and finishers are $1.25 \%, 1.10 \%$ and $1.00 \%$, respectively. Fernandes et al. (2009) and Ruiz-Feria (2009) reported that inclusion of arginine in diet beyond NRC (1994) recommendation improved broiler productivity. Kidd et al. (2001) reported of enhancement in growth of broilers upon supplementation with $0.2 \%$ arginine (Arg) above NRC (1994) recommendation. Various researches laying credence to the positive impact of Arg supplementation, have made members of the industry supplement broiler chicks Arg levels exceeding NRC (1994) recommended levels. Aside from Arg promoting the growth of chicken, several studies have reported on its role on the immune system of animals (Kwak et al., 1999; Kidd et al., 2001; Ochoa et al., 2001; Ruiz-Feria et al., 2001; de Jonge et al., 2002; Munir et al., 2009; Tan et al., 2009 b; Perez-Carbajal et al., 2010; Masoud et al., 2014).

Dietary arginine can be broken down to get molecules such as polyamines, nitric oxides and creatine. This presents great benefits to animals by protecting intestinal health. One other important function of arginine in diets is the alleviation of damage to the villus, dilation of crypt and depletion of goblet cells influenced by coccidia in broilers (Tan et al., 2014; Yin et al., 2014). Eimeria species is the causative organism for coccidiosis in chicken (Vermeulen et al., 2001). Their activities lead to several damages among the flock ranging from stress in intestine, diarrhea, malabsorption of nutrients and destruction to the epithelial cells (Kidd et al., 2001; Perez-Carbajal et al., 2010; Persia et al., 2006; Metzler-Zebeli et al., 2009).

In broiler chicken challenged with necrotic enteritis, supplementation of diet with arginine suppresses the growth of Clostridium perfringens which is the causative organism (Zhang et al., 2017). Arginine performs the role of substrate during biological production of many substances like glutamine, protein, glutamate, ornithine, nitric oxide, polyamines and proline (Khajali and Wideman, 2010). In other experiments carried out on porcine, arginine was found to regulate how metabolic genes for fat in skeletal muscles and adipose tissues were expressed (Tan et al., 2011). Arginine also provided an increase in muscles and reduction of the mass of body fat in grower and finisher pigs (Tan et al., 2009 b). Chen et al. (2011) reported a rise in growth performance of broilers when arginine was used in diets. There was also an improvement 
in the characteristics of the carcass and yield of meat-type ducks (Wu et al., 2011). Researches conducted by Khajali et al. (2011) and Jiao et al. (2010) on arginine supplementation revealed a reduction in the weight of leg muscle of broilers when the diet was deficient in Arg. Arginine has also been found to promote the health and reproductive performance of farm animals (Uni and Ferket, 2003; Wu, 2009; Ren et al., 2012). There was an influence on the various blood parameters in laying hens and broilers (Al-Hassani, 2011; Al-Daraji and Salih, 2012) upon supplementation of diets with Arg. It improved the immune system by helping the development of lymphoid organs, functioning of the spleen and thymus (Bistrain, 2004; Stechmiller et al., 2005). An experiment conducted by Kwak et al. (1999) reported weight loss of thymus, spleen and bursa of fabricius in chicks fed diet deficient in arginine.

The intestinal environment and gut microflora serve important functions in improving the health of broilers (Ziegler et al., 2013). Ren et al. (2014 a) reported of the production of nitric oxide by arginine which helped in destroying pathogenic microorganisms, promoted innate immunity and prevented the multiplication of disease causing micro-organisms in the intestine (Allen, 1999; Li et al., 2007). Allen (1999) researched on the importance of arginine in reducing the adverse effects of Eimeria tenella in chickens upon the administration of $500 \mathrm{mg} \mathrm{Arg} / \mathrm{kg}$. The addition of Arg in broiler diets increases the production of lymphocytes that fight infectious bursal disease virus in chickens (Tayade et al., 2006 a). The review focuses on arginine in disease prevention, growth, the immune system and its relationship to the gut microbiota of broiler chicken.

\section{Arginine and microbiota of poultry}

The intestinal microbiota refers to several and intricate combination of different substances originating from the alimentary and endogenous parts together with living microorganisms that make up the gut lumen (Hamer et al., 2011; Wylie et al., 2012). The count of bacteria living in the small intestine increases towards the gut end with the highest density living in the large intestine. Gill et al. (2006) reported that the microbiota residing in the colon represent as much as $1013-1014{\text { cells } \mathrm{g}^{-1}}^{-}$ of the luminal content and belongs to several groups of bacteria. Among the roles micro-organisms play in the host physiology and metabolism are luminal breakdown of non-digested compounds from the alimentary canal and production of vitamins $\mathrm{B}$ and $\mathrm{K}$. They also perform roles in the breakdown of some micronutrients, endogenous compounds (bile acids), fight against pathogens and regulate the immune system (O’Keefe, 2008; Round and Mazmanian, 2009; Laparra and Sanz, 2010).

The chicken intestine consists of several micro-organisms which adapt to a particular section. There are different environmental conditions pertaining to the different sections of the gut. The dominant micro-organisms in the crop and the small intestine are Lactobacillus spp., Enterococcus spp. and Streptococcus spp. (Lu et al., 2003; Apajalahti and Kettunen, 2006; Abbas et al., 2007). For every organism to realize its full potential there must be homeostasis. To maintain a very fit and efficient microbial population, conditions such as prevailing $\mathrm{pH}$, suitable growth substrates and the secretion of antibacterial by the host should be considered. The role of nutrition includes providing protection from immune diseases, enhancing the microbial 
population in the gut and improving hormonal environment (Humphrey and Klasing, 2004; Klasing, 2007). Harley and Prescott (1998) observed a rise in the count of Lactobacillus in the small intestine of turkey when arginine level increased to $1.0 \mathrm{~g} / \mathrm{kg}$ from 0 and concluded that it was an indication of improved gut health. Lactobacillus helps in the fermentation of carbohydrates to produce hydrogen peroxide and lactic acid which are considered to be the normal composition of the intestinal tract of chicken (Harley and Prescott, 1998).

The $\mathrm{pH}$ of the gut is decreased resulting from the production of lactic acid. This prevents the proliferation of several harmful bacteria like the enteropathogens, mycobacteria and Clostridium spp. There was a decrease in the number of Salmonella when the diet of murine was supplemented with arginine and this was attributed to nitric oxide produced following the supplementation of arginine (Eriksson et al., 2003). Nitric oxide is a powerful agent which has the ability of limiting the growth of harmful bacteria like Salmonella and other parasites living within the cells (Eriksson et al., 2003). Several researches that seek to identify the arginine pathway producing nitric oxide reported that there was a great increase in the macrophages produced after arginine supplementation which helped in providing defence against attack (Xia et al., 1996; Sung et al., 1991; Luiking et al., 2012). The increase of non-pathogenic bacteria helps in health promotion by boosting host immune defence, in mucin synthesis and increase in the number of epithelial cells.

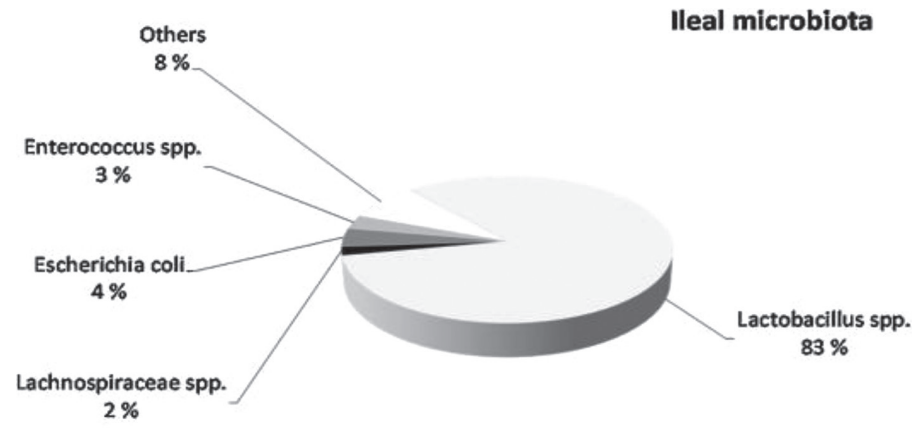

Caecal microbiota

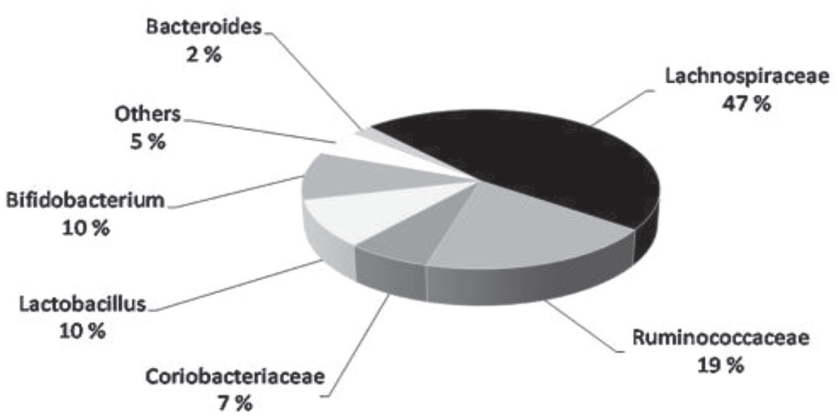

Figure 1. Ileum and caecum microbiota composition of 10 European farms (Alimetrics Ltd laboratory, unpublished results) 
Several factors affect the gut microbial environment of poultry. Notable amongst them are age, genotype, infections, immune response and diet (Luo et al., 2017; Aguzey et al., 2018). Diet, however, accounts for about 57\% while genetic factors account for $12 \%$ (Tomasello et al., 2014). The content of the microbial environment can be changed when there are changes in dietary composition (David et al., 2014). There are several properties of arginine that are beneficial to animals in preventing diseases such as infectious bursal disease, coccidiosis and necrotic enteritis of poultry (Amin et al., 2002; Tan et al., 2014, 2015). The composition of microbiota in the ileum and caecum of broilers is shown in Figure 1.

\section{Role of arginine on the immune system of poultry}

The largest immune organ which is the intestine, plays crucial roles in ensuring immunity of animals. The mucosa, apart from its functions of digestion and absorption, plays a key role in serving as an innate defence barrier against pathogens (Shao et al., 2013). The mucosa helps in health promotion and growth performance of chicken (Zavarize et al., 2012). It includes immune cells, cytokines and lymphoid tissues (Wershil and Furuta, 2008). Gao et al. (2017) reported that in ovo supplementation of arginine increased the weight of lymphoid organs (spleen, thymus, bursa of fabricius). The increase in weight of these organs was associated with an increase in immune functions (Gao et al., 2017).

One major challenge militating against the achievement of optimum growth rate and feed efficiency in poultry production is the incidence of diseases. Farm animals are usually confronted with various microbial challenges leading to poor performance because of the loss of dietary nutrients. This leads to serious losses in broiler production (Klasing et al., 2007). An important way of preventing and controlling disease is by improving host immune defences through nutrition. Among the various amino acids, arginine leads in immunomodulation (Jahanian, 2009; Tan et al., 2009 a; Ren et al., 2014 a). During investigations, it was found that arginine pathway produces NO (Kidd et al., 2001; Jahanian, 2009) because of infection, making it part of the inflammatory responses due to it being initiated by inflammatory cytokines (Allen, 1999). Ren et al. (2014 b) reported that dietary arginine exerts beneficial effects on the bacterial population in the jejunum.

There are two main ways by which farm animals develop resistance or fight diseases. These are the use of innate response which deals with the fast identification and possible ways of eliminating pathogenic organisms invading the host and adaptive response. Developing and maintaining of innate immune function is vital for animals, most especially poultry. Supplementation of arginine in the diet enhances both innate and adaptive immunity (Bronte and Zanovello, 2005) of farm animals. Several researchers have reported on the importance of Arg as an immune-boosting dietary supplement for birds (Evoy et al., 1998; Abdukalykova et al., 2008) and its inflammation relieving property (Coburn et al., 2012). One important molecule that is activated during innate immune response is cytokine. They are those proteins or peptides that transfer messages between cells (Guo et al., 2015). They play crucial roles in immunomodulation, protein mediation and physiological processes (Ma et al., 2007; Guo et al., 2015). Example of cytokines like IL-2 activates natural killer 
and $\mathrm{T}$ cytolytic cells, in charge of cellular immune responses in poultry (Yu et al., 2000). Lymphocytes are one subtype of white blood cells in vertebrate immune system. They are made up of natural killer cells performing cell-mediation roles and cytotoxic innate immunity. T cells are responsible for cell-mediation, cytotoxic adaptive immunity and B cells for humoral, antibody-driven adaptive immunity. Their importance in helping promote good health in an organism cannot be underestimated.

Several research works have underscored the importance of arginine in helping the development of lymphocytes and thereby promoting the work of the immune system in various models which are under immunological challenge (Abdukalykova et al., 2008; Li et al., 2007; Munir et al., 2009). Dietert and Austic (1994) reported that when diets were deficient in arginine supplementation, there was a reduction in the lymphocyte population and animals became susceptible to infections. Feeding broiler chicken an increasing dietary arginine level increases the weight of the thymus (Kwak et al., 1999). Arginine supplementation has effect on immunomodulation in animals by promoting response from lymphocyte to mitogens such as phytohemagglutinin and concanavalin A (Ochoa et al., 2001), enhancing the weight and work of the thymus gland and production of immune cells ( $\mathrm{Wu}, 2009)$.

Arginine supplementation increased the antibody titers to Newcastle, an acute and highly contagious disease with high morbidity and mortality (Kidd et al., 2001). It also increased the serum haptoglobin in porcine (Tan et al., 2009 a; Yang et al., 2012). Arginine is responsible for maintenance of mucosal integrity through improvement in the growth and development of enterocytes (Rhoads et al., 2008). Dietary arginine above the level recommended activate innate immunity through various signaling pathways thus promoting immune system functions ( $\mathrm{Li}$ et al., 2007; Wu et al., 2009). Guo et al. (2017) reported on modulation of immune system after arginine supplementation activated NO secretion, IL-2 and iNOS expression. During immunological response, the amount of arginine needed had been found to be greater than for growth and feed efficiency (Jahanian, 2009).

To maintain homeostasis in the gastrointestinal tract, there must be an increase in sIgA which is the first line of defence against disease causing organisms and enteric toxins. Arginine is known to increase sIgA content in small intestine (Mantis et al., 2011; Gao et al., 2017). Several researches on the dosage of Arg needed to enhance the immune system have been reported. Gao et al. (2017) reported that to facilitate improvement in intestinal health and overall immunity of chicken, in ovo feeding of $0.6 \mathrm{~mL}$ of $10 \mathrm{~g} / \mathrm{L}(\mathrm{w} / \mathrm{v})$ arginine solution at $17.5 \mathrm{~d}$ was recommended. Jahanian (2009) reported that for the optimum immune function of broiler chicks to be reached, arginine supplementation of 7\% above the NRC (1994) recommendation was required. Guo et al. (2015) and Xu et al. (2018) suggested that 3.56\% of arginine in diet was ideal for optimization of immune potential of broiler chicks. Zhang et al. (2018) reported that arginine supplementation in chicken diet at $0.3 \%$ lessened gut injury and normalized the population of microbiota in the ileum of chicken challenged with $C$. perfringens. 


\section{Arginine and its role in the prevention of poultry diseases}

The onset of some parasitic and infectious diseases reduces productivity and causes a great loss in the industry. Among the economically important poultry diseases reported worldwide are coccidiosis and Newcastle disease (Dalloul and Lillehoj, 2006). Dalloul and Lillehoj (2006) and De Gussem (2007) reported that coccidiosis alone accounted for a total loss of 3.2 billion USD annually to the poultry sector with the greater part of the loss resulting from the cost of diagnosis and treatment (Vermeulen et al., 2001). Arginine possesses several characteristics that make it provide immunity to chicken when supplemented in their diet. The role arginine plays in preventing some selected diseases of poultry are looked at briefly.

\section{Necrotic enteritis}

The causative organism of necrotic enteritis, Clostridium perfringens, is a gram positive and an anaerobic bacteria which resides in litter, soil or dust (Van Immerseel et al., 2009). Its symptoms include diarrhea, wet litter, lesions on the liver and incidence of mortality. It causes a reduction in growth, feed efficiency, digestion and absorption. This is as result of the damage to the intestine which normally characterizes the acute phase of the disease (Williams, 2005). Several research works have reported that in chicken challenged with necrotic enteritis, the walls of the small intestines become thinner, appearance of small reddish petechiae and hyperemia (Van Immerseel et al., 2009; Dahiya et al., 2005). It normally becomes pronounced by day 35 in the growth of broilers with severity of the clinical signs being age dependent.

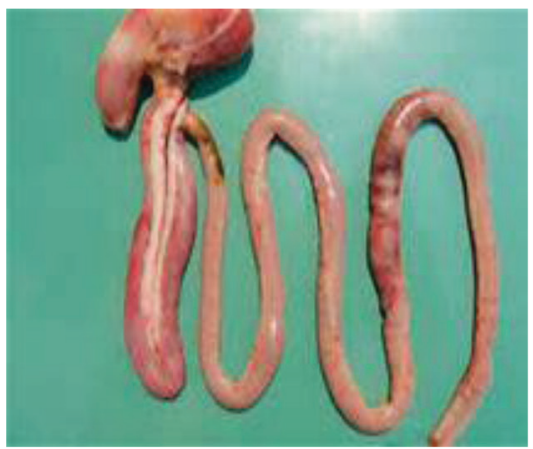

(A)

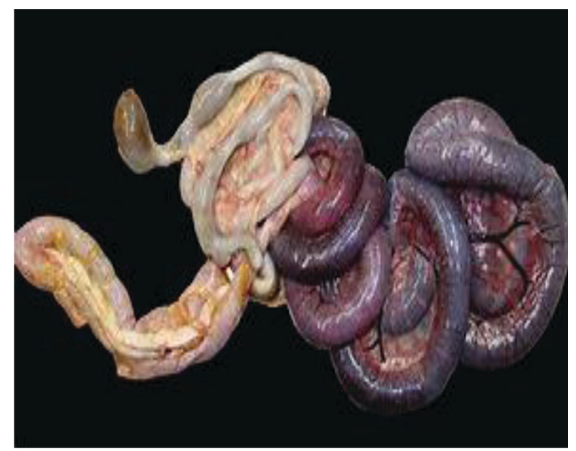

(B)

A. The onset of necrotic enteritis

B. The advanced stage of necrotic enteritis

Figure 2. Images of chicken intestines infected with necrotic enteritis (internet source)

With the ban on antibiotic use as growth promoters in broiler production, cases of necrotic enteritis have been on the rise with an estimated global loss of over 2 bil- 
lion dollars annually (Caly et al., 2015). Certain husbandry and dietary factors can lead to the multiplication of the causative organism leading to the onset of necrotic enteritis. A change in diet formulation, infections and lack of homeostasis can lead to incidence ofnecrotic enteritis. When broiler chickens are supplemented with arginine in their diet there is a marked improvement in innate immune response, absorption capacity of the intestine and the population of beneficial microbiome (Efron and Barbul, 1998; Evoy et al., 1998). Zhang et al. (2017) reported on birds challenged with necrotic enteritis, that arginine supplementation prevented the takeover of C. perfringens. Dietary supplementation with arginine also increased the nutrient absorption by enhancing the height of villi and preserving the gut morphology. Damages caused to the intestines of chicken infected with necrotic enteritis are shown in Figure 2 .

\section{Infectious bursal disease}

The infectious bursal disease (IBD) has the infectious bursal disease virus (IBDV) as its causative organism. It normally attacks chicks between 3 and 6 weeks of age and causes severe economic loss in the poultry sector resulting from the high mortality associated with its infection (Van Den Berg, 2000). It is also known as Gumboro disease with characteristic effect on immunosuppression and mortality. During infection it predisposes birds to other diseases and also interferes with effective vaccination leading to severe mortality (Sharma, 2000). The onset of the disease is sudden and may wipe out a whole farm within weeks after infection which is via oro-fecal route. Clinical signs include swollen feces stained vent, ruffled feathers, watery diarrhea, dehydration and birds lying prostrate.

Several factors account for mortality rate: previous immunity, strain, challenged dose, presence of concurrent disease and the ability of the flocks to produce a response. The greatest threat posed by infectious bursal disease is immunosuppression of chicks below three weeks of age where the signs are subclinical but may lead to chicks having bursal atrophy with cystic follicles and lymphocytopenia which renders chicks more susceptible to infections by agents that would not usually cause disease in immunocompetent birds (Tan et al., 2015). Arginine supplementation above the NRC recommended level boosts the immune system of poultry, leading to birds becoming resistant to IBD (Ruiz-Feria and Abdukalykova, 2009). Arginine prevents immunosuppression which brings about a reduction in the effectiveness of vaccines as a result of lysis of B cells and their precursors (Tayade et al., 2006 b; Emadi et al., 2011). Figure 3 shows images of the bursa affected by the disease. Arginine has the ability to stimulate into action different cell types like $\mathrm{T}$ and $\mathrm{B}$ cells, natural killer (NK) cells, lymphokine activated killer cells and macrophages (de Jonge et al., 2002; Tayade et al., 2006 a). de Jonge et al. (2002) reported that arginine supplementation increased the antibody titres and it is able to help in the differentiation of pro-B to pre-B cells. Arginine performs strong immunoregulatory function on the immune system and enhances specific immune response against IBD (Tayade et al., 2006 a). 


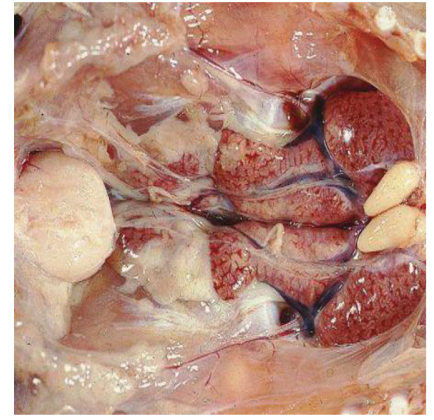

( A )

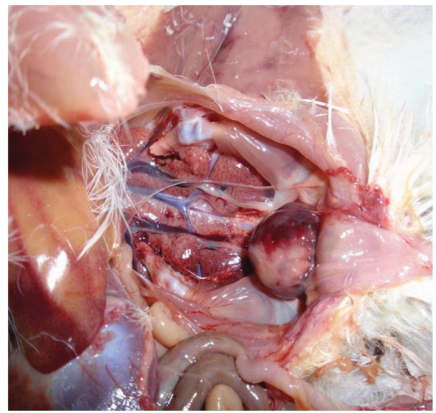

( B )

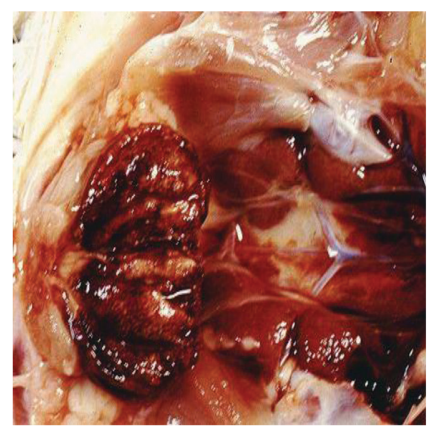

(C)

A. The onset of the disease with swollen bursa

B. Blood stained bursa

C. A fully infected bursa dissected

Figure 3. Images of infectious bursal disease of poultry (internet source)

\section{Coccidiosis}

Coccidiosis, an economically significant disease affects farm animals including poultry. Its causative organism is a coccidian protozoa with the intestinal tract being the most affected. It is spread through contact. Signs such as bloody diarrhea and loss of appetite characterize coccidiosis infection. Chicks with compromised immune system suffer severe symptoms with high mortality rate (Tan et al., 2014). Coccidiosis negatively affects the height of villus, crypt depth, ratio between villus height and crypt depth. Laika and Jahanian (2017) reported that arginine supplementation of $110 \%$ exceeding the recommended level ameliorated the adverse effect of coccidiosis on the intestines. This was attributed to the production of NO by the activities of synthase on Arg (Moncada and Higgs, 1993; Khajali et al., 2011). Other researches attributed it to the fact that $\mathrm{NO}$ becomes toxic to coccidia because it reacts with intracellular iron-containing compounds (Moncada et al., 1991; Ovington and Smith, 1992). Arginine supplementation also promotes intestinal growth (Yao et al., 
2011; He et al., 2011). Figure 4 shows images of the caeca of poultry affected by coccidiosis.

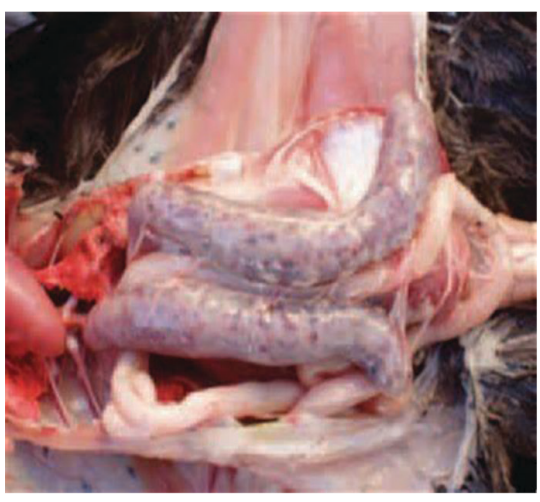

(A)

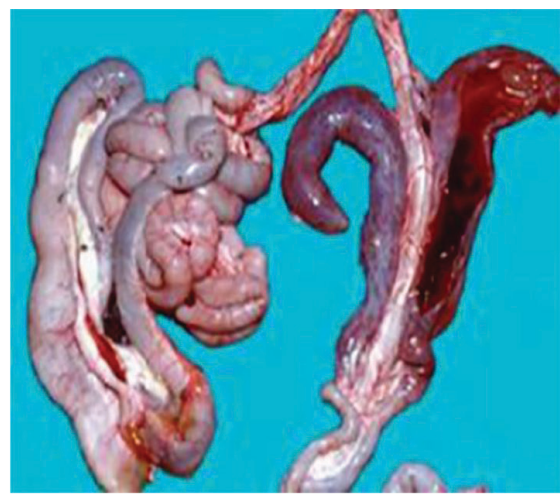

(B)

A. The caecum of chicken affected by coccidiosis

B. Caecum dissected showing blood stains

Figure 4. The caeca of chicken infected by coccidiosis (internet source)

\section{How arginine promotes growth}

There has been numerous research works on how amino acid supplementation impacts on growth of poultry. Diets deficient in arginine which is an important amino acid have been found to cause a reduction in feed intake and adversely affect weight gain (Kwak et al., 1999). Arginine improves growth performance of animals by two possible ways: enhancing protein synthesis, inhibition of proteolysis promoting whole body growth and serving as secretagogue; causing the release of prolactin, glucagon, insulin, GH, IGF-1 and preventing hypothalamic SS release which result in GH release in the blood stream, increasing protein synthesis (Hanew, 2000; Newsholme et al., 2005; Yao et al., 2008).

Diets lacking arginine lead to low feed intake resulting in a decrease in body weight, slow growth rate and disabilities (Kwak et al., 1999). Arginine supplied above the NRC (1994) recommended level enhanced productivity of broilers (Corzo et al., 2003; Fernandes et al., 2009; Ruiz-Feria, 2009). The level of supplementation of arginine beyond the NRC (1994) recommendations however varies among different researchers. Supplementing the diet with $0.2 \%$ arginine above the NRC (1994) requirement resulted in improved growth performance (Kidd et al., 2001). Fernandes et al. (2009) reported that Arg supplementation of $1.39 \%, 1.49 \%, 1.59 \%, 1.69 \%$ and $1.79 \%$ in the starter diet of $0-21 \mathrm{~d}$ broilers caused an increase in breast weight and fillet thickness. Ebrahimi et al. (2014) reported on the increase in weight and feed efficiency of broiler chicken after being fed high concentrations of digestible arginine at concentrations of $2.2 \%$ for $0-10 \mathrm{~d}, 2.0 \%$ for $11-24 \mathrm{~d}$ and $1.85 \%$ for $25-46 \mathrm{~d}$. Xu et al. (2018) also reported that digestible Arg concentration of $1.69-2.14 \%$ for $0-21 \mathrm{~d}$ 
and $1.53-1.98 \%$ for $21-42$ d) increased growth performance of chicken. The combined effect of arginine supplementation on chicken is shown in Figure 5.

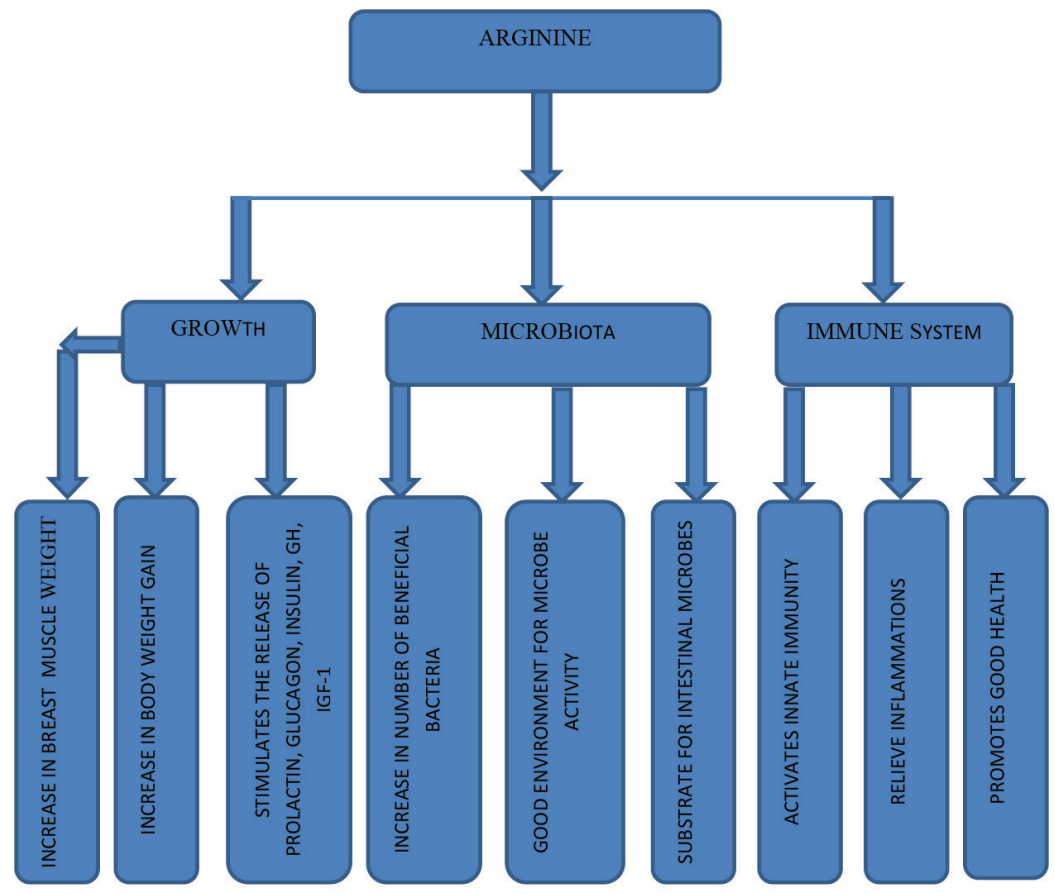

Figure 5. Effects of arginine supplementation on growth, microbiota and the immune system of broiler chicken

\section{Conclusion}

Arginine supplementation increased serum levels of GH, IGF-1 and insulin (Xu et al 2018). From literature, it is evident that arginine supplementation to the diet of chicken improved growth performance, fight against the onset of pathogenic diseases by helping boost the immune functions and promoting the growth of beneficial microbes in the gut. The use of arginine in the diet of broiler chicken has been shown to prevent diseases such as necrotic enteritis, infectious bursal disease and coccidiosis by promoting intestinal health, prevent immunosuppression and increase dietary intake. Different levels have different effect on broiler chicken. Further research is needed to determine the best level for broiler production.

\section{References}

A b ba s H., Hilmi A., S u rakk a J., A paja la ht i P.E.J. (2007). Identification of the most abundant Lactobacillus species in the crop of 1- and 5-week-old broiler chickens. Appl. Environ. Microb., 73: 7867-7873. 
A bdukalykova S.T., Z h a o X., R u i z-F e ri a C.A. (2008). Arginine and vitamin E modulate the subpopulations of T lymphocytes in broiler chickens. Poultry Sci., 87: 50-55.

A gu z e y H.A., G a o Z., W u H., Ch en g G. (2018). Influence of feed form and particle size on gizzard, intestinal morphology and microbiota composition of broiler chicken. Poultry Fish Wild Sci., 6: 196.

A l - D a r a j i H.J., S a lih A.M. (2012). The influence of dietary arginine supplementation on blood traits of broiler chickens. Pak. J. Nutr., 11: 258-264.

A 1 - H a s s a n i A.S. (2011). Effect of dietary supplementation with different levels of arginine on some blood traits of laying hens. Intern. J. Poult. Sci., 10: 705-709.

A 11 e n P.C. (1999). Effects of daily oral doses of L-arginine on coccidiosis infections in chickens. Poultry Sci., 78: 1506-1509.

A min H.J., Z a mora S.A., McMillan D.D., Fick G.H., Butzner J.D., Parsons H.G. (2002). Arginine supplementation prevents necrotizing enterocolitis in the premature infant. J. Pediatr., 140: 425-431.

A paja lahti J., Kettunen A. (2006). Microbes of the chicken gastrointestinal tract. In: Avian gut function in health and disease, Perry G.C. (ed.). Poultry Sci. Symp. Series, 28, CABI Publishing, Wallingford, pp. 113-121.

B a 11 R.O., U r s c h e 1 K.L., P e n c h a r z P.B. (2007). Nutritional consequences of interspecies differences in arginine and lysine metabolism. J. Nutr., 137: 1626S-1641S.

B istrain B.R. (2004). Practical recommendations for immune-enhancing diets. J. Nutr., 134: 2868-2872.

B r o n t e V., Z a n o ve 11 o P. (2005). Regulation of immune responses by L-arginine metabolism. Nat. Rev. Immunol., 5: 641-654.

Caly D.L., D'Inca R., A u clair E., Drider D. (2015). Alternatives to antibiotics to prevent necrotic enteritis in broiler chickens: a microbiologist's perspective. Front. Microb., 6: 1336.

Che n J., Wa n g M., K on g Y., M a H., Z o u S. (2011). Comparison of the novel compounds creatine and pyruvate on lipid and protein metabolism in broiler chickens. Animal, 5: 1082-1089.

C oburn L.A., Gong X., S ing h K., A s i m M., S c u 11 B.P., A 11 a m a n M.M., Willi a m s C.S., Rosen M.J., Washington M.K., Barry D.P., Piazuelo M.B., Casero R.A., Ch a turvedi R., Zhao Z., Wilson K.T. (2012). L-arginine supplementation improves responses to injury and inflammation in dextran sulfate sodium colitis. PLoS ONE, 7: e33546.

Corzo A., Moran Jr. E.T., H o e hler D. (2003). Arginine needs of heavy broiler males: applying the ideal protein concept. Poultry Sci., 82: 402-407.

Dahi y a J.P., Ho ehle r D., Wilki e D.C., Van Kesse 1 A.G., Drew M.D. (2005). Dietary glycine concentration affects intestinal Clostridium perfringens and lactobacilli populations in broiler chickens. Poultry Sci., 84: 1875-1885.

D a 11 o u 1 R.A., L i 11 e h oj H.S. (2006). Poultry coccidiosis: recent advancements in control measures and vaccine development. Expert Rev. Vaccines, 5: 143-163.

D a vi d L.A., M a u r i c e C.F., C a r m o d y R.N., G o o t e n berg D.B., B u t to n J.E., Wolfe B.E. (2014). Diet rapidly and reproducibly alters the human gut microbiome. Nature, 505: 559-563.

D e Gus s e m M. (2007). Coccidiosis in poultry: Review on diagnosis, control, prevention and interaction with overall gut health. Proc. 16th European Symposium on Poultry Nutrition, pp. 253-261.

De Jonge W.J., Kwikkers K.L., te Velde A.A., van Deventer S.J.H., Nolte M.A., M e bius R.E., Ruijter J.M., L a mers M.C., L a mers W.H. (2002). Arginine deficiency affects early B cell maturation and lymphoid organ development in transgenic mice. J. Clin. Invest., 110: $1539-1548$.

D i e t e r t R.R., A u s t i c R.E. (1994). Environment-immune interactions. Poultry Sci., 73: 1062-1076.

Ebrahimi M., Shahneh A.Z., Shivazad M., Pirsaraei Z.A., Tebianian M., Ruiz- Feria C.A., Adibmoradi M., Nourijelyani K., Mohamadnejad F. (2014). The effect of feeding excess arginine on lipogenic gene expression and growth performance in broilers. Brit. Poultry Sci., 55: 81-88.

E fron D.T., B a r b u 1 A. (1998). Modulation of inflammation and immunity by arginine supplements. Curr. Opin. Clin. Nutr. Metab. Care, 1: 531-538.

Emadi M., Jahanshiri F., Kaveh K., Hair-Bejo M., Ideris A., Alimon A.R. (2011). Nutrition and immunity: The effects of the combination of arginine and tryptophan on growth per- 
formance, serum parameters and immune response in broiler chickens challenged with infectious bursal disease vaccine. Avian Pathol., 40: 63-72.

Erik s s on S., Ch a m b e r s B.J., R h e n M. (2003). Nitric oxide produced by murine dendritic cells is cytotoxic for intracellular Salmonella enterica sv. Typhimurium. Scand. J. Immunol., 58: 493-502.

Ev o y D., L i e b e r m a n M.D., F a h e y T.J., D a ly J.M. (1998). Immunonutrition: The role of arginine. Nutrition, 14: 611-617.

F a c k e $1 \mathrm{~m}$ a y e r F.O. (2005). Protein arginine methyltransferases: guardians of the Arg? Trends Biochem. Sci., 30: 666-671.

Fernandes J.I.M., Murakami A.E., Martins E.N., Sakamoto M.I., Garcia E.R.M. (2009). Effect of arginine on the development of the pectoralis muscle and the diameter and the protein: deoxyribonucleic acid rate of its skeletal myofibers in broilers. Poultry Sci., 88: 1399-1406.

G a o T., Z ha o M.M., Z hang L., L i J.L., Yu L.L., L v P.A., G a o F., Z hou G.H. (2017). Effects of in ovo feeding of L-arginine on the development of lymphoid organs and small intestinal immune barrier function in posthatch broilers. Anim. Feed Sci. Technol., 225: 8-19.

Gill S.R., Pop M., Deboy R.T., Eckburg B., Turnbaugh P.J., Samuel B.S. (2006). Metagenomic analysis of the human distal gut microbiome. Science, 312: 1355-1359.

G u o Y.W., S h i B.L., Yan S.M., X u Y.Q., L i J.L., L i T.Y. (2015). Effects of arginine on cytokines and nitric oxide synthesis in broilers. J. Anim. Plant. Sci., 25: 366-371.

H a m e r H.M., D e Preter D., W in dey K., Verbeke K. (2011). Functional analysis of colonic bacterial metabolism: relevant to health. Am. J. Physiol. Gastr. L., 302: G1-G9.

$\mathrm{H}$ a n e w K. (2000). The mechanism of arginine- and insulin-induced GH release in humans. Endocrinol. J., 47: 23-27.

H a r l e y J.P., P r e s c o t t L.M. (1998). Laboratory exercises in microbiology (2nd ed.). W.M.C. Brown Publishers, New York, US.

He Q., Tang H., Ren P., Kong X., Wu G., Y in Y., Wang Y. (2011). Dietary supplementation with L-arginine partially counteracts serum metabonome induced by weaning stress in piglets. J. Proteome Res., 10: 5214-5221.

H u m phrey B.D., K l a s ing K.C. (2004). Modulation of nutrient metabolism and homeostasis by the immune system. World Poultry Sci. J., 60: 90-100.

$\mathrm{J}$ a h a n i a n R. (2009). Immunological responses as affected by dietary protein and arginine concentrations in starting broiler chicks. Poultry Sci., 88: 1818-1824.

Ji a o P., Gu o Y., Yang X., L ong F. (2010). Effects of dietary arginine and methionine levels on broiler carcass traits and meat quality. J. Anim. Vet. Adv., 9: 1546-1551.

K h a j a li F., Wi d e m a n R.F. (2010). Dietary arginine: metabolic, environmental, immunological and physiological interrelationships. World Poultry Sci. J., 66: 751-766.

Khajali F., Tahma sebi M., Has san pour H., Akbari M.R., Qujeq D., Wideman R.F. (2011). Effects of supplementation of canola meal-based diets with arginine on performance, plasma nitric oxide, and carcass characteristics of broiler chickens grown at high altitude. Poultry Sci., 90: 2287-2294.

K i d d M.T., P e e b le s E.D., W h it m a r s h S.K., Ye a t m a n J.B., W i d e m a n R.F. (2001). Growth and immunity of broiler chicks as affected by dietary arginine. Poultry Sci., 80: 1535-1542.

Kim S.W., McPherson R.L., Wu G. (2004). Dietary arginine supplementation enhances the growth of milk-fed young pigs. J. Nutr., 134: 625-630.

K 1 a s ing K.C. (2007). Nutrition and the immune system. Brit. Poultry Sci., 48: 525-537.

K w a k H., A u s t i c R.E., D i e t e r t R.R. (1999). Influence of dietary arginine concentration on lymphoid organ growth in chickens. Poultry Sci., 78: 1536-154.

L a b a d a n M.C., H s u K.N., A u s t i c R.E. (2001). Lysine and arginine requirements of broiler chickens at two- to three-week intervals to eight weeks of age. Poultry Sci., 80: 599-606.

Laika M., Jahanian R. (2017). Increase in dietary arginine level could ameliorate detrimental impacts of coccidial infection in broiler chickens. Livest. Sci., 195: 38-44.

L a p a r r a J.M., S a n z Y. (2010). Interactions of gut microbiota with functional food components and nutraceuticals. Pharmacol. Res., 61: 219-225.

L e w is S. (1996). Avian biochemistry and molecular biology. Cambridge University Press, New Jersey, New York City, USA. 
L i P., Y in Y.L., Li D., K i m S.W., Wu G. (2007). Amino acids and immune function. Brit. J. Nutr., 98: 237-252.

Liu D., Gu o S., Gu o Y. (2012 a). Xylanase supplementation to a wheatbased diet alleviated the intestinal mucosal barrier impairment of broiler chickens challenged by Clostridium perfringens. Avian Pathol., 41: 291-298.

L i u X., Wu X., Y in Y., Li u Y., Geng M., Yang H., Wu G. (2012 b). Effects of dietary L-arginine or N-carbamylglutamate supplementation during late gestation of sows on the miR-15b/16, miR-221/222, VEGFA and eNOS expression in umbilical vein. Amino Acids, 42: 2111-2119.

L u J., I d ri s U., H a r m on B., H o f a c r e C., M a u re r J.J., L e e M.D. (2003). Diversity and succession of the intestinal bacterial community of the maturing broiler chicken. Appl. Env. Microb., 69: 6816-6824.

Luiking Y.C., Ten Have G.A., Wolfe R.R., D e utz N.E. (2012). Arginine de novo and nitric oxide production in disease states. Am. J. Physiol. Endocrinol. Metab., 303: e1177-1189.

L u o Y., Zh an g L., L i H., S m idt H., Wrigh t A.G., Z hang K. (2017). Different types of dietary fibers trigger specific alterations in composition and predicted functions of colonic bacterial communities in BALB/c Mice. Front. Microbiol., 8: 966.

Ma C.S., Nichols K.E., Tangye S.G. (2007). Regulation of cellular and humoral immune responses by the SLAM and SAP families of molecules. Annu. Rev. Immunol., 25: 337-379.

Ma n t is N.J., R o l N., C o r thé s y B. (2011). Secretory IgA's complex roles in immunity and mucosal homeostasis in the gut mucosal. Immunology, 4: 603-611.

Masoud A., Marziyeh E., Ahmad Z.S., Mahmoud S., Zarbakht A.P., Majid T., K e r a m a t Y.Q. (2014). The effects of L-arginine on growth, small intestine, and immune system of broilers in starter period. Annu. New York Acad. Sci., 521: 72-87.

Metzler-Zebeli B.U., Eklund M., Mosenthin R. (2009). Impact of osmoregulatory and methyl donor functions of betaine on intestinal health and performance in poultry. World Poultry Sci. J., 65: 419-442.

Moncada S., Higg s A. (1993). The L-arginine-nitric oxide pathway. New Engl. J. Med., 329: 2002-2012.

M o n c a d a S., P a $1 \mathrm{~m}$ e r R.M.J., H i g g s E.A. (1991). Nitric oxide: physiology, pathophysiology and pharmacology. Pharmacol. Rev., 43: 109-142.

Mountzouris K.C., Tsirtsikos P., Kalamara E., Nitsch S., Schatzmayr G., F e g e r o s K. (2007). Evaluation of the efficacy of a probiotic containing Lactobacillus, Bifidobacterium, Enterococcus, and Pediococcus strains in promoting broiler performance and modulating cecal microflora composition and metabolic activities. Poultry Sci., 86: 309-317.

Munir K., Muneer M.A., Masaoud E., Tiwari A., Mahmud A., Chaudhry R.M., R a s hid A. (2009). Dietary arginine stimulates humoral and cell-mediated immunity in chickens vaccinated and challenged against hydropericardium syndrome virus. Poultry Sci., 88: 1629-1638.

Newsholme P., Brennan L., Rubi B., Maechler P. (2005). New insights into amino acid metabolism, $\beta$-cell function and diabetes. Clin. Sci., 108: 185-194.

NRC (1994). Nutrient Requirements of Poultry, 9th ed. National Academy Press, Washington, DC, USA.

O ' K e e fe S.J. (2008). Nutrition and colonic health: the critical role of the microbiota. Curr. Opin. Gastroen., 24: 51-58.

Ochoa J.B., Strange J., Kearney P., Gellin G., Endean E., Fitzpatrick E. (2001). Effects of L-arginine on the proliferation of T lymphocyte subpopulations. J.-Parenter. Enter. Nutr., 25: 23-29.

Ovington K.S., S m ith N.C. (1992). Cytokines, free radicals and resistance to Eimeria. Parasitol. Today, 8: 422-426.

Perez-Carbaja 1 C., Caldwe 11 D., F arne 11 M., String fellow K., P oh 1 S., Cas co G., Pro-Martine z A., Ru i z-F e r i a C.A. (2010). Immune response of broiler chickens fed different levels of arginine and vitamin E to a coccidiosis vaccine and Eimeria challenge. Poultry Sci., 89: 1870-1877.

P er s i a M.E., Young E.L., U t t e rb a c k P.L., P a r s on s C.M. (2006). Effects of dietary ingredients and Eimeria acervulina infection on chick performance, apparent metabolizable energy, and amino acid digestibility. Poultry Sci., 85: 48-55. 
Ravindran V. (2016). Feed-induced specific ileal endogenous amino acid losses: measurement and significance in the protein nutrition of monogastric animals. Anim. Feed Sci. Technol., 221: 304-313.

Ren W., Y in Y., Li u G., Yu X., L i Y., Yang G., Li T., Wu G. (2012). Effect of dietary arginine supplementation on reproductive performance of mice with porcine circovirus type 2 (PCV2) infection. Amino Acids, 42: 2089-2094.

Ren W., Chen S., Y in J., Du an J., Li T., Li u G., F eng Z., Tan B., Y in Y., Wu G. (2014 a). Dietary arginine supplementation of mice alters the microbial population and activates intestinal innate immunity. J. Nutr., 166: 988-995.

R en W., Y in J., Wu M., Li u G., Yang G., Xi on Y., S u D., Wu L., Li T., Chen S., Du an J., Y in Y., Wu G. (2014 b). Serum amino acids profile and the beneficial effects of L-arginine or L-glutamine supplementation in Dextran Sulfate Sodium Colitis. PLoS One, 9: e88335.

Rhoads J.M., Liu Y., Niu X., Surendran S., Wu G. (2008). Arginine stimulates cdx2-transformed intestinal epithelial cell migration via a mechanism requiring both nitric oxide and phosphorylation of p70 S6 kinase. J. Nutr., 138: 1652-1657.

R ound J.L., Mazmanian S.K. (2009). The gut microbiota shapes intestinal immune responses during health and disease. Nat. Rev. Immunol., 9: 313-323.

R u i z - F e r i a C.A. (2009). Concurrent supplementation of arginine, vitamin E, and vitamin C improve cardiopulmonary performance in broilers chickens. Poultry Sci., 88: 526-535.

R u i z - F e ri a C.A., K i d d M.T., W i d e m a n R.F. (2001). Plasma levels of arginine, ornithine, and urea and growth performance of broilers fed supplemental L-arginine during cool temperature exposure. Poultry Sci., 80: 358-369.

Ruiz-Feria C.A., Abduka lyk ova S.T. (2009). Arginine and vitamin E improve the antibody response to infectious bursal disease virus (IBDV) and sheep red blood cells in broiler chickens. Brit. Poultry Sci., 50: 291-297.

S h a o Y., Gu o Y., Wang Z. (2013). $\beta$-1, 3/1, 6-glucan alleviated intestinal mucosal barrier impairment of broiler chickens challenged with Salmonella enterica serovar Typhimurium. Poultry Sci., 92: $1764-1773$.

S h a r m a J.M., K i m I.J., R a u t e n s c h 1 e i n S., Y e h H-Y. (2000). Infectious bursal disease virus of chickens: Pathogenesis and immunosuppression. Dev. Comp. Immunol., 24: 223-235.

Stechmiller J.K., Langkamp-Henken B., Childress B., Herrlinger-Garcia K.A., Hudgens B., Ti an J. (2005). Arginine supplementation does not enhance serum nitric oxide levels in elderly nursing home residents with pressure ulcers. Biol. Res. Nurs., 6: 289-299.

S ung Y.J., H o t chkis s J.H., A u s t i c R.E., D i e te r t R.R. (1991). L-arginine dependent production of a reactive nitrogen intermediate by macrophages of a uricotelic species. J. Leukocyte Biol., 50: 49-56.

Tan B., Li X.G., Kong X., Huang R., Ruan Z., Yao K., D eng Z., Xie M., Shinzato I., Y in Y., W u G. (2009 a). Dietary L-arginine supplementation enhances the immune status in earlyweaned piglets. Amino Acids, 37: 323-331.

Tan B., Yin Y., Liu Z., Li X., Xu H., Kong X., Huang R., Tang W., Shinzato I., S m ith S., Wu G. (2009 b). Dietary L-arginine supplementation increases muscle gain and reduces body fat mass in growing-finishing pigs. Amino Acids, 37: 169-175.

Tan B.E., Y in Y.L., Li u Z.Q., Tang W.J., Xu H.J., Konga X.F., Li X.G., Yao K., Gu W., S m ith S.B., Wu G. (2011). Dietary L-arginine supplementation differentially regulates expression of fat-metabolic genes in porcine adipose tissue and skeletal muscle. J. Nutr. Biochem., 22: $441-445$.

Tan J., A p plegate T.J., Li u S., Gu o Y., Ei c he r S.D. (2014). Supplemental dietary L-arginine attenuates intestinal mucosal disruption during coccidial vaccine challenge in broiler chickens. Brit. J. Nutr., 112:1098-109.

Ta n J.Z., Gu o Y.M., A p p l e g a te T.J., D u E.C., Z h a o X. (2015). Dietary L-arginine modulates immunosuppression in broilers inoculated with an intermediate strain of infectious bursa disease virus. J. Sci. Food Agric., 95: 126-135.

Tay a de C., Ja is w a 1 T., M is hra S., K ot i M. (2006 a). L-arginine stimulates immune response in chickens immunized with intermediate plus strain of infectious bursal disease vaccine. Vaccine, 24: $552-560$. 
Tay a d e C., K ot i M., M is hra S.C. (2006 b). L-arginine stimulates intestinal intraepithelial lymphocyte functions and immune response in chickens orally immunized with live intermediate plus strain of infectious bursal disease vaccine. Vaccine, 24: 5473-5480.

Tomas ello G., Tralongo P., Damiani P., S in a gra E., Di Trapani B., Zeenny M.N. (2014). Dismicrobism in inflammatory bowel disease and colorectal cancer: changes in response of colocytes. World J. Gastroenterol., 20: 18121-18130.

Un i Z., F e r ke t P. (2003). Enhancement of development of oviparous species by in ovo feeding. U.S. Regular Patent US 6,592,878 B2, Washington, DC., US.

Van Den B erg T.P. (2000). Acute infectious bursal disease in poultry: A review. Avian Pathol., 29: $175-194$.

Van Im m e r s e e 1 F., R o o d J.I., M o or e R.J., T i t b a 11 R.W. (2009). Rethinking our understanding of the pathogenesis of necrotic enteritis in chickens. Trends Microbiol., 17: 32-36.

Ver m e u l e n A.N., S c h a a D.C., S c het ters T.M. (2001). Control of coccidiosis in chickens by vaccination. Vet. Parasitol., 100: 13-20.

Wers hil B.K., Furut a G.T. (2008). 4: Gastrointestinal mucosal immunity. J. Allergy Clin. Immunol., 121: S380-S383.

W i 11 i a m s R.B. (2005). Intercurrent coccidiosis and necrotic enteritis of chickens: rational, integrated disease management by maintenance of gut integrity. Avian Pathol., 34: 159-180.

W u G. (2009). Amino acids: metabolism, functions, and nutrition. Amino Acids, 37: 1-17.

W u L.Y., F a n g Y.J., Gu o X.Y. (2011). Dietary L-arginine supplementation beneficially regulates body fat deposition of meat-type ducks. Brit. Poultry Sci., 52: 221-226.

Wu X., Wu Y.L., Y in Y.Q., Liu X.D., Liu Z.Q., Liu T.J., Li R., Huang L., Ruan Z., D e $\mathrm{n} g$ Z. (2012). Effect of dietary arginine and N-carbamoylglutamate supplementation reproduction and gene expression of eNOS, VEGFA and PlGF1 in on in late pregnancy of sow placenta. Anim. Reprod. Sci., 132: 187-192.

Wyli e K.M., Truty R.M., Sharpton T.J., Mihindukulasuriya K.A., Zhou Y., Gao H. (2012). Novel bacterial taxa in the human microbiome. PLoS ONE, 7: e35294.

X i a Y., D a w s o n V.L., D a w s o n T.M., S n y d e r S.H., Z w e i e r J.L. (1996). Nitric oxide synthase generates superoxide and nitric oxide in arginine-depleted cells leading to peroxynitrite-mediated cellular injury. Proc. Natl. Acad. Sci., 93: 6770-6774.

X u S., L in Y., Z eng D., Z h o u M., Z eng Y., Wang H. (2018). Bacillus licheniformis normalize the ileum microbiota of chickens infected with necrotic enteritis. Sci. Rep., 8: 17-44.

Yang H., L in B.Q., Z hang L., Wang T., D u H.J. (2012). Effects of L-arginine and lactobacillus on growth performance, blood biochemical indexes and intestinal mucosal morphology of weanling piglets (in Chinese). J. Fujian Agric. Forestry Univ. (Nat. Sci. Ed.), 4: 515-519.

Ya o K., Y in Y.L., Chu W.Y., Li u Z.Q., D eng D., Li T.J., H u a n g R.L., Zhang J.S., Tan B., Wang W.C., Wu G.Y. (2008). Dietary arginine supplementation increases mTOR signaling activity in skeletal muscle of neonatal pigs. J. Nutr., 138: 867-872.

Y a o K., Gu an S., Li T., H u a n g R., W u G., R u a n Z., Y in Y. (2011). Dietary L-arginine supplementation enhances intestinal development and expression of vascular endothelial growth factor in weanling piglets. Brit. J. Nutr., 105: 703-709.

Y in J., Ren W., Duan J., Wu L., Chen S., Li T., Y in Y., Wu G. (2014). Dietary arginine supplementation enhances intestinal expression of SLC7A7 and SLC7A1 and ameliorates growth depression in mycotoxin challenged pigs. Amino Acids, 46: 883-892.

Yu T.K., C a u d e 11 E.G., S m i d C., G r i m m E.A. (2000). IL-2 activation of NK cells: involvement of MKK1/2/ERK but not p38 kinase pathway. J. Immunol., 164: 6244-6251.

Zavarize K.C., S artori J.R., Gonzales E., Pezzato A.C. (2012). Morphological changes of the intestinal mucosa of broilers and layers as affected by fasting before sample collection. Rev. Bras. Cienc. Avic., 14: 2002-2012.

Zhang B., Lv Z., Li H., Gu o S., Li u D., Gu o Y. (2017). Dietary L-arginine inhibits intestinal Clostridium perfringens colonisation and attenuates intestinal mucosal injury in broiler chickens. Brit. J. Nutr., 118: 321-332.

Zhang B., Lv Z., Li Z., Wang W., Li G., Guo Y. (2018). Dietary L-arginine supplementation alleviates the intestinal injury and modulates the gut microbiota in broiler chickens challenged by Clostridium perfringens. Front. Microbiol., 9: 1716. 
Zi e gle r T.R., Evan s M.E., F ernande z- Est ivari z C., Jones D.P. (2013). Trophic and cytoprotective nutrition for intestinal adaptation, mucosal repair, and barrier function. Annu. Rev. Nutr., 23: $229-261$.

Received: 3 IX 2019

Accepted: 5 XI 2019 